\title{
Recent methodologies for reliability-based design optimization
}

\author{
G. Kharmanda ${ }^{1,3, a}$, A. Mohsine ${ }^{2,3}$, A. Makloufi ${ }^{3}$ and A. El-Hami ${ }^{3}$ \\ 1 Faculty of Mechanical Engineering, Aleppo University, Syrian Arab Republic \\ 2 INSA de Lyon, Lab. d'Automatique Industrielle, Bât. St Exupéry, 25, Avenue Jean Capelle, 69621 Villeurbanne, France \\ 3 INSA de Rouen, LMR, BP 08, Avenue de l’Université 76801 St Etienne du Rouvray, France
}

Received 31 May 2007, accepted 11 January 2008

\begin{abstract}
In the field of Deterministic Design Optimization (DDO), the designer reduces the structural cost without taking into account uncertainties concerning materials, geometry and loading. This way the resulting optimum solution may represent a lower level of reliability and thus a higher risk of failure. But in the Reliability-Based Design Optimization (RBDO) model, the mean values of uncertain system variables are usually used as design variables, and the cost is optimized subject to prescribed probabilistic constraints as defined by a nonlinear mathematical programming problem. Therefore, a RBDO solution that reduces the structural weight in uncritical regions does not only provide an improved design but also a higher level of confidence in the design. In this paper, we present the advantage of the DDO and RBDO models and next some recent methodologies in order to show that the RBDO model is a practical tool for structural engineers.
\end{abstract}

Key words: Reliability-based design optimization; structural reliability; probabilistic model

\section{Nomenclature}

$\begin{array}{lll}\mathbf{x} & : & \text { Deterministic variable } \\ \mathbf{y} & : & \text { Random variable } \\ \mathbf{u} & : & \text { Normalized variable } \\ \mathbf{m}_{i} & : & \text { Mean of random variable } \\ \sigma_{\mathrm{i}} & : & \text { Standard-deviation of random variable } \\ \beta_{t} & : & \text { Target reliability (safety) index } \\ \beta(\mathbf{x}, \mathbf{u}) & : & \text { Reliability (safety) index in } u \text {-space } \\ d_{\beta}(\mathbf{x}, \mathbf{y}) & : & \text { Reliability (safety) index in } x y \text {-space } \\ g_{k}(\mathbf{x}) \leqslant 0 & : & \text { Geometrical, physical, functional } \\ H(\mathbf{x}, \mathbf{u}) \leqslant 0 & : & \text { Limit state in u-space } \\ G(\mathbf{x}, \mathbf{y}) \leqslant 0 & : & \text { Limit state in xy-space }\end{array}$

\section{Introduction}

In the last 40 years, there has been extensive research focused on structural optimization with dynamic constraint because the response of a structure to dynamic excitation depends, to a large extent, on the first few natural frequencies of the structure. Excessive vibration occurs when the frequency of the dynamic excitation is close to one of the natural frequencies of the structure. The optimum design of structures with frequency constraints is of great

\footnotetext{
a Corresponding author:

mgk@scs-net.org; amohsine@gmail.com;

amaklouf@insa-rouen.fr; aelhami@insa-rouen.fr
}

importance, particularly in the aeronautical and automotive industries. In designing most structures, it is often necessary to restrict the fundamental frequency or several of the lower frequencies of the structure to a prescribed range in order to avoid severe vibration. However, the designer reduces the structural cost without taking into account uncertainties concerning material properties, geometric dimension and loading. This way the resulting optimal design may represent a lower level of reliability and thus a higher risk of failure. Since structural problems are non-deterministic, it is clear that the introduction of the reliability concept plays an important role in the structural optimization field. Deterministic design optimization enhanced by reliability performances and formulated within the probabilistic framework is called ReliabilityBased Design Optimization (RBDO). The RBDO problem is often formulated as a minimization of the initial structural cost under constraints imposed on the values of elemental reliability indices corresponding to various limit states (Madsen and Friis Hansen [13]; Kleiber et al. [11]). The objective of the RBDO model is to design structures which should be both economic and reliable where the solution reduces the structural weight in uncritical regions. It does not only provide an improved design but also a higher level of confidence in the design. The classical approach (Feng and Moses [3]) can be carried out in two separate spaces: the physical space and the normalized space. Since very many repeated searches are needed in the above two spaces, the computational time 


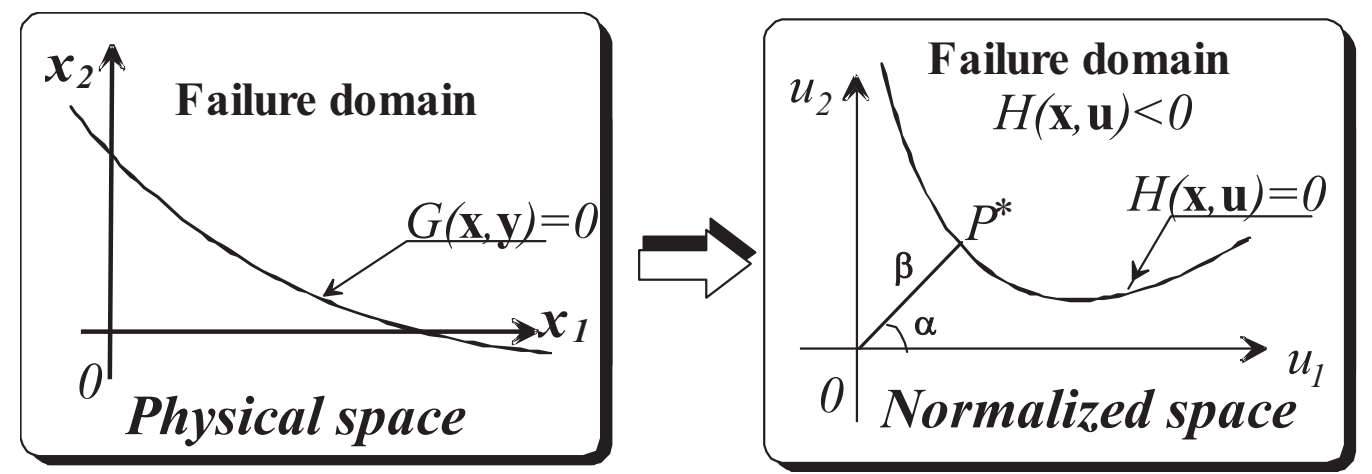

Fig. 1. The transformation between the physical space and normalized one.

for such an optimization is a big problem. To overcome these difficulties, two points of view have been considered. From reliability view point, RBDO involves the evaluation of probabilistic constraints, which can be executed in two different ways: either using the Reliability Index Approach (RIA), or the Performance Measure Approach (PMA) (Tu et al. [20]; Youn et al. [22]). Recently, the enhanced hybrid mean value $(\mathrm{HMV}+)$ method is proposed by Youn et al. [23], to improve numerical stability and efficiency in the Most Probable Point (MPP) search. The major difficulty lies in the evaluation of the probabilistic constraints, which is prohibitively expensive and even diverges with many applications. However, from optimization view point, an efficient method called the Hybrid Method (HM) has been elaborated by Kharmanda et al. [6] where the optimization process is carried out in a Hybrid Design Space (HDS). This method has been shown to verify the optimality conditions relative to the classical RBDO method. The advantage of the hybrid method allows us to satisfy a required reliability level for different cases (static, dynamic, ...), but the vector of variables here contains both deterministic and random variables. The hybrid RBDO problem is thus more complex than that of deterministic design. The major difficulty lies in the evaluation of the structural reliability, which is carried out by a special optimization procedure. Next, an Optimum Safety Factor (OSF) methodology has been proposed by Kharmanda et al. (2004) to simplify the optimization problem (reduction of number of variables) and aims to find at least a local optimum solution because it is based on the optimality condition. However, this method cannot be used in some dynamic cases. For example, in the freely vibrating structures, if the failure interval $[f a, f b]$ is given, we cannot determine the reliabilitybased optimum solution using the optimum safety factor method. So Mohsine et al. [15, 16] proposed to develop the hybrid method with object of improving the optimum value of the objective function. The proposed method is called the Improved Hybrid Method (IHM). This method has been also shown to verify the optimality conditions relative to the classical RBDO method. We note that the OSF method can provide a big part of the RBDO problem analytically. So it reduces efficiently the computing time of the problem relative to the hybrid and the improved hybrid methods. In this paper, a new methodology called Safest Point method (SP) is presented as a conjoint to the OSF method in order to solve the freely vibrating structures.

\section{Reliability analysis}

Structural reliability analysis is a tool that assists the design engineer to take into account all possible uncertainties during the design and construction phases and the lifetime of a structure in order to estimate the probabilities of failure $P_{f}$. The vector of deterministic variables $\mathbf{x}$ to be used in the system design and optimization, the uncertainties are modeled by a vector of stochastic physical variables affecting the failure scenario. The knowledge of these variables is not, at best, more than statistical information and we admit a representation in the form of random variables. For a given design rule, the basic random variables are defined by their joint probability distribution associated with some expected parameters; the vector of random variables is denoted herein $\mathbf{Y}$ whose realizations are written $\mathbf{y}$. The safety is the state in which the structure is able to fulfill all the functioning requirements (e.g. strength and serviceability) for which it is designed. To evaluate the failure probability with respect to a chosen failure scenario, a limit state function $G(\mathbf{x}, \mathbf{y})$ is defined by the condition of good functioning of the structure. In Figure 1, the limit between the state of failure $G(\mathbf{x}, \mathbf{y})<0$ and the state of safety $G(\mathbf{x}, \mathbf{y})>0$ is known as the limit state (failure) surface $G(\mathbf{x}, \mathbf{y})=0$. The failure probability is then calculated by:

$$
P_{f}=P_{r}[G(\mathbf{x}, \mathbf{y}) \leqslant 0]=\int_{G(\mathbf{x}, \mathbf{y}) \leqslant 0} f_{\mathbf{y}}(\mathbf{y}) d y_{1} \ldots d y_{n}
$$

This multi-dimensional integral becomes very expensive to compute when the dimension of $\mathbf{X}$ is large. For these reasons, the First and the Second Order Reliability Methods FORM/SORM (Ditlevsen and Madsen [2]) have been developed, these methods are based on calculation of most probable point. A convenient way to represent reliability 
is by a reliability index, $\beta$ which can be defined as:

$$
\beta=-\Phi^{-1}\left(P_{f}\right)
$$

where $\Phi$ is the standard Gaussian cumulated function. In Figure 1b, the Most Probable Point (MPP) of failure can be found in a standard normalized space, $\mathbf{U}$, for a single hard or failure driven constraint $G(\mathbf{x}, \mathbf{y})$. The components of $\mathbf{U}$ are normally distributed with zero means and unit variance and are statistically independent. Any set of continuous random variables, $\mathbf{X}$, can be transformed to $\mathbf{U}$ using an one-to-one transformation i.e. $\mathbf{u}=T(\mathbf{x}, \mathbf{y})$, where $\mathbf{Y}$ are distribution parameters of $\mathbf{X}$. Many such transformations are possible, but the one typically used is called the Rosenblatt transformation (1976). The MPP, $\mathbf{u}^{*}$, lies on the failure surface or the limit state surface $G(\mathbf{x}, \mathbf{y})=H(\mathbf{x}, \mathbf{u})=0$, and its location is the closest point on the limit state surface to the origin in U-space. The MPP can be found by solving the following constrained optimization.

$$
\beta=\min _{\mathbf{u}}\left(\mathbf{u}^{T} \mathbf{u}=\min \sqrt{\sum_{1}^{m} u_{j}^{2}}\right)
$$

where the standard space, the limit state function takes the form $H(\mathbf{x}, \mathbf{u})=G(\mathbf{x}, \mathbf{y})$.

For more details, the interested reader can the different works of Hasofer and Lind [5], Liu and Der Kiureghian [12] and Ditlevsen and Madsen [2].

\section{Deterministic design optimization}

In Deterministic Design Optimization (DDO), the system safety may be taken into account by assigning safety factors to certain structural parameters. Using these safety factors, the optimization problem which is carried out in the physical space (Fig. 1a), consists in minimizing an objective function (cost, volume of material, ... ) subject to geometrical, physical or functional constraints in the form

$$
\begin{aligned}
& \min _{\mathbf{x}}: f(\mathbf{x}) \\
& \text { subject to: } g_{k}(\mathbf{x}) \leqslant 0, k=1, \ldots, K
\end{aligned}
$$

where $\mathbf{x}$ designates the vector of deterministic design variables. The values of the proposed safety factors principally depend on the engineering experience, but, when designing a new structure, we cannot pre-determine the real critical points, and the choice of these coefficients may therefore be wrong. Suitable geometry, material properties and loads are assumed, and an analysis is then performed to provide a detailed behavior of the structure. However, changes of the loads, variability of material properties, and uncertainties regarding the analytical models all contribute to the probability that the structure does not perform as intended. To address this concern, analysis methods have been developed to deal with the statistical nature of the input information. As structures are becoming still more complex (e.g. space shuttle engine components, space structures, advanced tactical fighters, etc.) and performance requirements are becoming more ambitious, the need for analyzing the influence of uncertainties and computation of reliability has been growing.

Over the last ten years there has been an increasing trend in analyzing structures using probabilistic information on loads, geometry, material properties, and boundary conditions. In order to evaluate the structural safety level (see problem (3)), a reliability analysis must be carried out without taking into account the safety factor from problem (4). After having followed the Deterministic Design Optimization (DDO) procedure by a reliability analysis, we can distinguish between two cases:

Case 1. High reliability level: when choosing high values of safety factors for certain parameters, the structural cost (or weight) will be significantly increased because the reliability level becomes much higher than the required level for the structure. So, the design is safe but very expensive.

Case 2. Low reliability level: when choosing small values of safety factors or bad distribution of these factors, the structural reliability level may be too low to be appropriate. For example, Grandhi and Wang [4] found that the resulting reliability index of the optimum deterministic design of a gas turbine blade is $\beta=0.0053$ under some uncertainties. This result indicated that the reliability at the deterministic optimum is quite low and needs to be improved by probabilistic design.

For both cases, we can find that there is a strong need to integrate the reliability analysis in the optimization process in order to control the reliability level and to minimize the structural cost or weight in the non-critical regions of the structure. In the next section, we show how this can be performed efficiently. The integration of reliability analysis into engineering design optimization is termed Reliability-Based Design Optimization (RBDO). Numerical applications in Section 5, shows the advantage of the RBDO procedure relative to DDO one and the efficiency of the RBDO methods for different analysis cases (static, modal, transient).

\section{Reliability-based design optimization}

In the literature, many studies are reported in the field of RBDO. To the best of our knowledge, Stevenson [19] was the first who introduced reliability analysis in connection with design optimization. On the one hand, structural reliability research concentrates on probabilistic descriptions of phenomena and application to code oriented safety design. On the other hand, optimization research works toward efficient algorithms for locating optima particularly in large-scale systems using prescribed deterministic constraints. In the design of structural members, it is possible to establish the connection between optimization and reliability. This approach can be unified in the sense that all the uncertain quantities can be modeled as random variables. Hence, a lot of numerical computation is required in the space of random variables in order 
to evaluate the system reliability. Furthermore, the optimization process itself is executed in the space of design variables that are deterministic. Consequently, in order to search for an optimum structure, the design variables are repeatedly changed, and each set of the design variables corresponds to a new random variable space which then needs to be manipulated to evaluate the structural reliability at that point. Since too many repeated searches are needed in the above two spaces, the computational time for such an optimization is a great problem.

Feng and Moses [3,14] attempted to unify these efforts. In this case, optimization procedures should explicitly consider safety either directly in its cost function or as one of its primary constraints. Next, the use of approximations in structural reliability and optimization in order to reduce the computer time was presented in several research papers. In the works of Chandu and Grandhi (1995) and Grandhi and Wang [4], an efficient reliability analysis based on approximating the limit state functions using two-point adaptive nonlinear approximation, has been developed to reduce the computational time. On the other hand, Madsen and Friis Hansen [13] integrated the expected failure cost in the objective function in order to compare it with a classical reliabilitybased optimization procedure, but this algorithm required a high number of iterations to converge. The computational cost of this combined approach is higher than that of the nested RBDO model, i.e., it requires about $50 \%$ more computational effort to converge as compared with the classical RBDO approach. Der Kiureghian and Polak [1] and Tu et al. [20] proposed several forms in order to satisfy the constraints and simplify the problem.

It is clear that efforts were directed towards the development of efficient techniques and general purpose programs to perform the reliability analysis. These programs and procedures compute the reliability index of a structure for a defined failure mode, but do not provide an optimum set of design parameters for improving the reliability of a structure for defined random information. Since the reliability index is computed iteratively, an enormous amount of computer time is involved in the whole design process. A Hybrid Method (HM) based on simultaneous solution of the reliability and the optimization problem has successfully reduced the computational time problem (Kharmanda et al. [6]). The HM allows us to satisfy a required reliability level, can be used in several cases (static, dynamic... ), may provide local optimal solutions and has a big number of optimization variables (deterministic and random). An Optimum Safety Factor (OSF) method has been elaborated (Kharmanda et al. 2004) to reduce the problem scale and to improve the optimum value of the objective function. The OSF method cannot be used in special case of dynamic studies. Therefore, an Improved Hybrid Method (IHM) has been developed by Mohsine et al. [16] which can improve the optimum value of the objective function relative to HM. Recently; we developed a very simple method, called Safest Point (SP) method which is conjoint to the OSF one to solve the special dynamic cases.

\subsection{Classical method (CM)}

Traditionally, for the reliability-based optimization procedure we use two spaces: the physical space and the normalized space (Fig. 1). Therefore, the reliability-based optimization is performed by nesting the two following problems:

\section{Optimization problem:}

$$
\begin{aligned}
& \min : f(\mathbf{x}) \\
& \text { subject to }: g_{k}(\mathbf{x}) \leqslant 0 \text { and } \beta(\mathbf{x}, \mathbf{u}) \geqslant \beta_{t}
\end{aligned}
$$

where $f(\mathbf{x})$ is the objective function, $g_{k}(\mathbf{x}) \leqslant 0$ are the associated constraints, $\beta(\mathbf{x}, \mathbf{u})$ is the reliability index of the structure, and $\beta_{t}$ is the target reliability.

2 Reliability analysis:

The reliability index $\beta(\mathbf{x}, \mathbf{u})$ is determined by solving the minimization problem:

$$
\begin{aligned}
& \beta=\min \operatorname{dis}(\mathbf{u})=\sqrt{\sum_{1}^{m} u_{j}^{2}} \\
& \text { subject to }: H(\mathbf{x}, \mathbf{u}) \leqslant 0
\end{aligned}
$$

where $\operatorname{dis}(\mathbf{u})$ is the distance in the normalized random space, given by:

$$
\operatorname{dis}=\sqrt{\sum u_{i}^{2}}
$$

and $H(\mathbf{x}, \mathbf{u})$ is the performance function (or limit state function) in the normalized space, defined such that $H$ (x, $\mathbf{u})<0$ implies failure, see Figure $1 \mathrm{~b}$. In the physical space, the image of $H(\mathbf{x}, \mathbf{u})$ is the limit state function $G(\mathbf{x}, \mathbf{y})$, see Figure 1. In general, when a probabilistic approach is used instead of a conventional deterministic approach, some of the uncertain quantities should be modelled as random variables. A lot of numerical calculations are required in the space of random variables to evaluate the system reliability. Furthermore, the optimization process itself is executed in the space of design variables which are deterministic. Consequently, in order to search for an optimal structure, the design variables are changed repeatedly, and each set of design variables corresponds to a new random variable space which then needs to be manipulated to evaluate the structural reliability at that point. Since a very large number of repeated searches are needed in the above two spaces, the computational time for such an optimization is a big problem. To reduce the effects of this difficulty, a hybrid method (HM) based on simultaneous solution of the reliability and the optimization problem has been elaborated (Kharmanda et al. 2001 and $[2])$.

\subsection{Hybrid method (HM)}

The solution of the above nested problems leads to very large computational time, especially for large-scale structures. In order to improve the numerical performance, the hybrid approach consists in minimizing a new form of the 


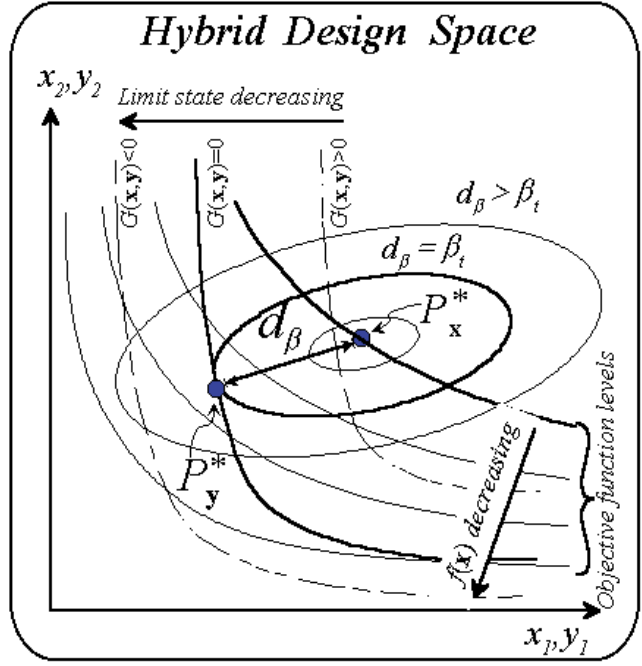

Fig. 2. Hybrid design space for normal distribution.

objective function $F(\mathbf{x}, \mathbf{y})$ subject to a limit state and to deterministic as well as to reliability constraints, as:

$$
\begin{aligned}
& \min _{\mathbf{x}, \mathbf{y}}: F(\mathbf{x}, \mathbf{y})=f(\mathbf{x}) \cdot d_{\beta}(\mathbf{x}, \mathbf{y}) \\
& \text { subject to }: G(\mathbf{x}, \mathbf{y}) \leqslant 0 \\
& \text { and } \quad: g_{k}(\mathbf{x}) \leqslant 0 \\
& \quad: d_{\beta}(\mathbf{x}, \mathbf{y}) \geqslant \beta_{t} .
\end{aligned}
$$

Here, $d_{\beta}(\mathbf{x}, \mathbf{y})$ is the distance in the hybrid space between the optimum and the design point, $d_{\beta}(\mathbf{x}, \mathbf{y})=\operatorname{dis}(\mathbf{u})$. The minimization of the function $F(\mathbf{x}, \mathbf{y})$ is carried out in the Hybrid Design Space (HDS) of deterministic variables $\mathbf{x}$ and random variables $\mathbf{y}$.

An example of this HDS is given in Figure 2, containing design and random variables, where the reliability levels $d_{\beta}$ can be represented by ellipses in case of normal distribution, the objective function levels are given by solid curves and the limit state function is represented by dashed level lines except for $G(\mathbf{x}, \mathbf{y})=0$. We can see two important points: the optimal solution $P_{x}^{*}$ and the reliability solution $P_{y}^{*}$ (i.e. the design point found on the curves $G(\mathbf{x}, \mathbf{y})=0$ and $\left.d_{\beta}=\beta_{t}\right)$.

In the paper of Kharmanda et al. [2], we had demonstrated that the HM reduced the computational time almost $80 \%$ relative to the classical RBDO approach. Using the HM, the optimization process is carried out in the Hybrid Design Space (HDS) where all numerical information about the optimization process can be modeled. Furthermore, the classical method (CM) has weak convergence stability because it is carried out in two spaces (physical and normalized spaces).

The efficiency of the hybrid approach allows us extending for solving more complex problems. When considering the standard-deviation values as variables, we can treat a big number of optimization variables (see [15]). For an industrial application, the HM has been efficiently applied on a lorry brake system to find the reliability-based optimum design of the front body shape on dynamic behavior (see [17]).

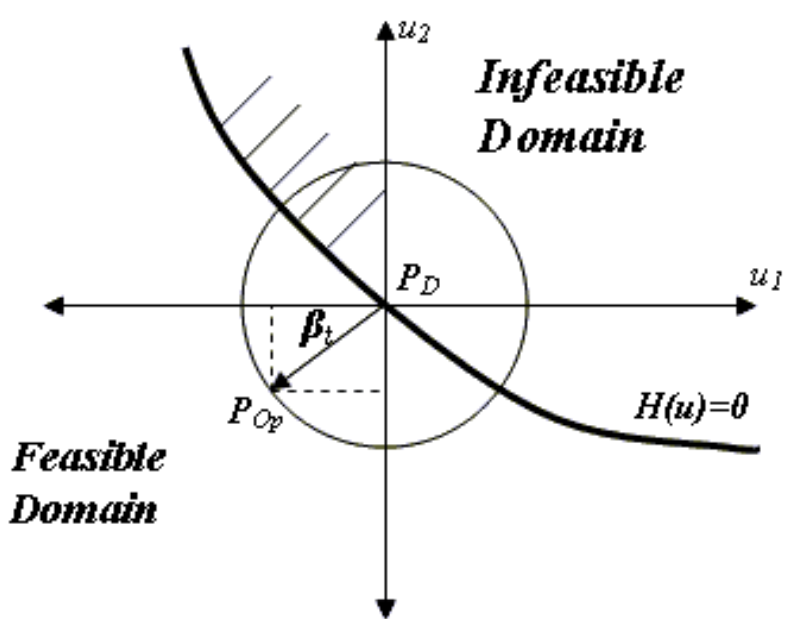

Fig. 3. Illustrated example for two-dimensional space.

\subsection{Optimum safety factor method (OSF)}

In fact, when using the HM, we have a complex optimization problem with many variables. Solving this problem, we get a local optimum. When changing the starting point, we may get another local optimum. This way the designer has to repeat the optimization process to get several local optima. However, to overcome these drawbacks, an optimum safety factor method has been proposed (Kharmanda et al. 2004). In general, when considering the normal distribution law, the normalized variable $u_{i}$ is given by:

$$
u_{i}=\frac{y_{i}-m_{i}}{\sigma_{i}}, i=1, \ldots, n .
$$

The standard deviation $\sigma_{i}$ can be related to the mean value $m_{i}$ by:

$$
\sigma_{i}=\gamma_{i} \cdot m_{i}, \text { or } \sigma_{i}=\gamma_{i} \cdot x_{i}, i=1, \ldots, n .
$$

This way we introduce the safety factors $S_{f_{i}}$ corresponding to the design variables $x_{i}$. The design point can be expressed by:

$$
y_{i}=S_{f_{i}} \cdot x_{i}, i=1, \ldots, n \text {. }
$$

For an assumed failure scenario $G(\mathbf{y})<0$, the equation of the optimum safety factor for a single limit state case can be written in the following form (see the developments in Kharmanda et al. 2004):

$$
S_{f_{i}}=1 \pm \gamma_{i} \cdot \beta_{t} \sqrt{\frac{\left|\frac{\partial G}{\partial y_{i}}\right|}{\sum_{i=1}^{n}\left|\frac{\partial G}{\partial y_{i}}\right|}}, i=1, \ldots, n
$$

Here, the $\operatorname{sign} \pm$ depends on the sign of the derivative, i.e.,

$$
\frac{\partial G}{\partial y_{i}}>0 \Leftrightarrow S_{f_{i}}>1, i=1, \ldots, n
$$




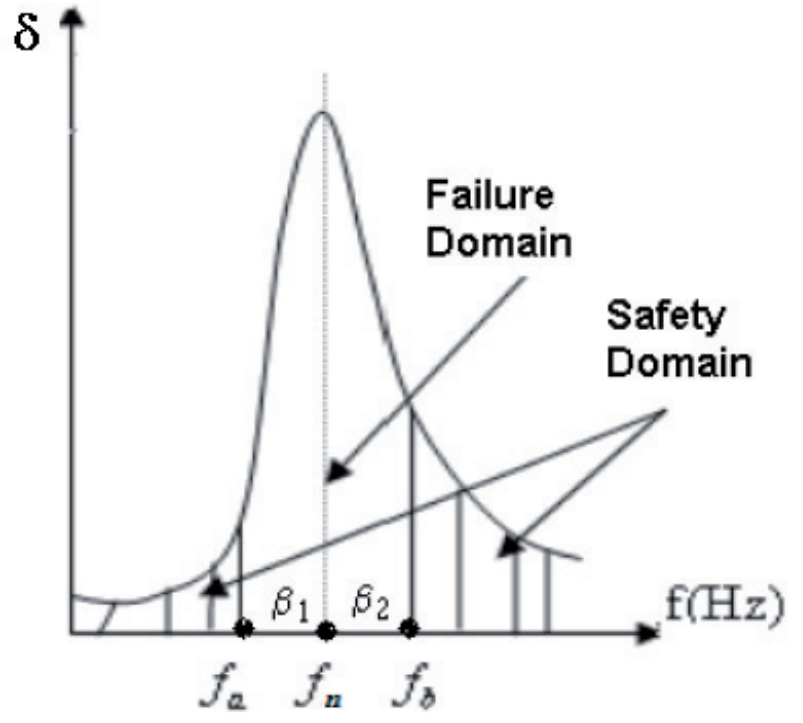

Fig. 4. The safest point at frequency $f n$.

$$
\frac{\partial G}{\partial y_{i}}<0 \Leftrightarrow S_{f_{i}}<1, i=1, \ldots, n .
$$

Let us consider an example of only two normalized variables $u_{1}$ and $u_{2}$ (see Fig. 3).

For an industrial application, Yang et al. [21] compared the results and efficiencies of different RBDO methods on an exhaust system. The objective was to minimize the weight of the system subject to constraints that the reliability of the resultant forces in each frequency region should be less than specified values. All in all 144 constraints were imposed, but many of them were inactive. After having tested several RBDO methods, they concluded that:

"Kharmanda et al. (2004) also used structural safety factors, based on the sensitivity of the limit-state function, for RBDO. In addition to its simplified computational framework to completely decouple the optimization and the reliability analyses, the method has two advantages:

1. It incorporates the partial safety-factor concept with which most designers are familiar. And, theoretically, safety factors do not have to be tied to the individual random variables and thus the MPPs (Most Probable Points).

2. It produces progressively improved reliable designs in the initial steps that help designers keep track of their designs."

According to this experience, this method is considered as a very good active constraint strategy (for problems with many constraints). A numerical application in Section 5.2 shows the advantage of the OSF procedure relative to HM on a tri-material beam under static distributed load.

\subsection{Improved hybrid method (IHM)}

Using the hybrid method, we can obtain local optima and designer may then select the best optimum. In the improved hybrid method, we introduce the design point and the optimum solution in the objective function and the constraints at the design point and at the optimum solution as follows:

$$
\begin{aligned}
\min _{\mathbf{x}, \mathbf{y}}: F(\mathbf{x}, \mathbf{y})=f(\mathbf{x}) \cdot d_{\beta}(\mathbf{x}, \mathbf{y}) \cdot f\left(\mathbf{m}_{y}\right) \\
\text { subject to } \quad: G(\mathbf{x}, \mathbf{y}) \leqslant 0 \\
\quad: g_{k}(\mathbf{x}) \leqslant 0 \\
\quad: g_{j}\left(\mathbf{m}_{y}\right) \leqslant 0 \\
\text { and } \quad: d_{\beta}(\mathbf{x}, \mathbf{y}) \geqslant \beta_{t} .
\end{aligned}
$$

The random vector $\mathbf{x}$ has mean values $\mathbf{m}_{y}$ and standarddeviations $\sigma_{y} . f\left(\mathbf{m}_{y}\right)$ is the optimal objective function and $g_{i}\left(\mathbf{m}_{y}\right)$ is the constraint at which we can control the optimal configuration. The solution of this problem depends on two important points. It can be carried out simultaneously in the hybrid design space (HDS).

We consider two models: the optimal model corresponding to $\mathbf{x}$ and the design one corresponding to $\mathbf{y}$ to be first evaluated. The functions $f(\mathbf{x}), f\left(\mathbf{m}_{y}\right)$ and $d_{\beta}(\mathbf{x}, \mathbf{y})$ are next calculated to get the value of the new objective function:

$$
F(\mathbf{x}, \mathbf{y})=f(\mathbf{x}) \cdot d_{\beta}(\mathbf{x}, \mathbf{y}) \cdot f\left(\mathbf{m}_{y}\right) .
$$

We estimate the different constraints to test the convergence. If the convergence is not verified, we update the vectors $x$ and $y$, re-evaluate the models, all functions until the convergence (see the developments in [16]). A numerical application in Section 5.3 shows the improved optimum objective produced by the IHM relative to HM on a triangular plate structure under dynamic behavior.

\subsection{Safest point method (SP)}

The reliability-based optimum structure under free vibrations for a given interval of eign-frequency is found at the safest position of this interval where the safest point has the same reliability index relative to both sides of the interval. A simple method has been proposed here to meet the safest point requirements relative to a given frequency interval.

Let consider a given interval $[f a, f b]$. For the first shape mode, to get the reliability-based optimum solution for a given interval, we consider the equality of the reliability indices:

$$
\beta_{a}=\beta_{b} \quad \text { or } \beta_{1}=\beta_{2}
$$

with

$$
\beta_{a}=\sqrt{\sum_{i=1}^{n} u_{i}^{a}} \text { and } \beta_{a}=\sqrt{\sum_{i=1}^{n} u_{i}^{b}} \quad i=1, \ldots, n .
$$

To verify the equality (16), we propose the equality of each term. So we have:

$$
u_{i}^{a}=-u_{i}^{b}, \quad i=1, \ldots, n .
$$



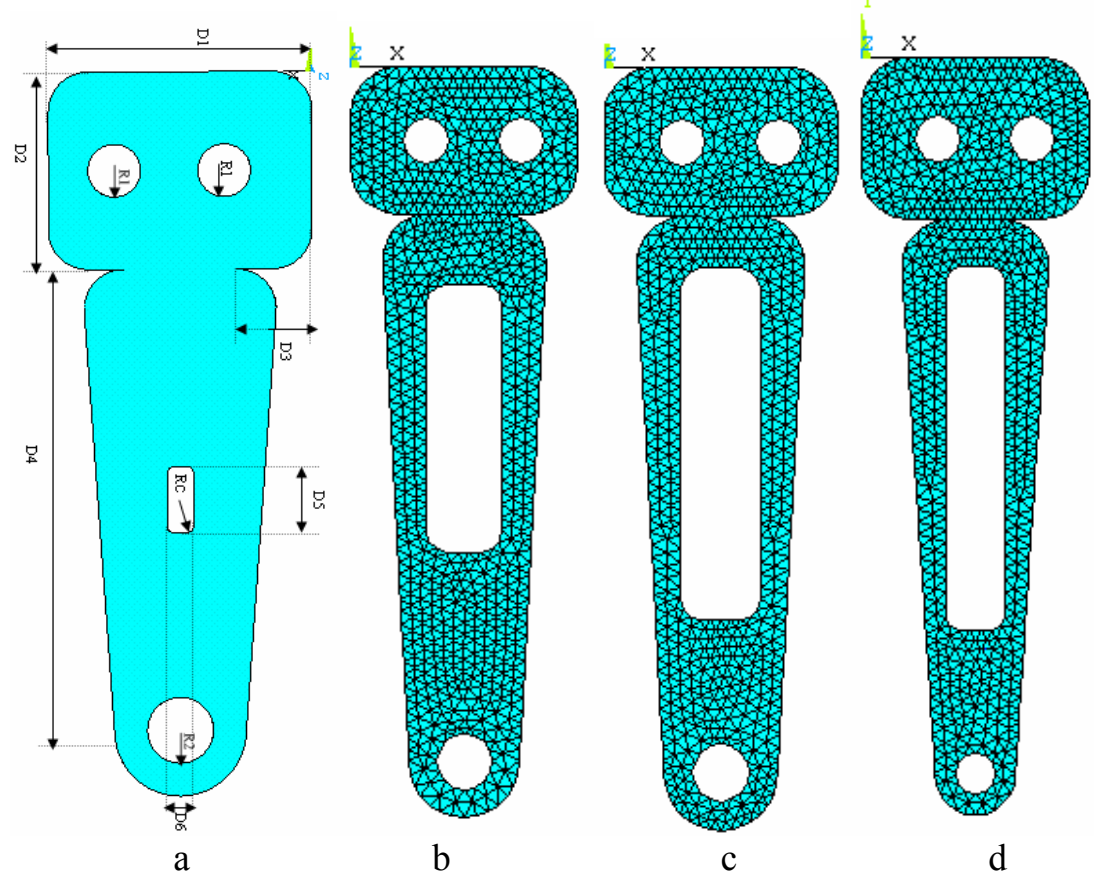

Fig. 5. (a) Geometrical models of the structure. (b), (c), (d) Different optima when safety factors $1.5,1.2$ and 1.1, respectively.

Table 1. Initial values parameter.

\begin{tabular}{|c|c|c|c|c|c|c|c|c|c|c|c|}
\hline & $\begin{array}{c}E \\
(\mathrm{MPa}) \\
\end{array}$ & $\begin{array}{c}\text { Density } \\
\left(\mathrm{kg} / \mathrm{mm}^{3}\right)\end{array}$ & $\begin{array}{c}\text { Poisson's } \\
\text { ratio }\end{array}$ & $\begin{array}{c}D 1 \\
(\mathrm{~mm}) \\
\end{array}$ & $\begin{array}{c}D 2 \\
(\mathrm{~mm}) \\
\end{array}$ & $\begin{array}{c}D 3 \\
(\mathrm{rad})\end{array}$ & $\begin{array}{c}D 4 \\
(\mathrm{rad})\end{array}$ & $\begin{array}{c}D 5 \\
(\mathrm{~mm}) \\
\end{array}$ & $\begin{array}{c}D 6 \\
(\mathrm{~mm}) \\
\end{array}$ & $\begin{array}{c}R c \\
(\mathrm{rad})\end{array}$ & $\begin{array}{c}R 0 \\
(\mathrm{~mm})\end{array}$ \\
\hline values & $2.1 \times 10^{6}$ & $78 \mathrm{e}-7$ & 0.3 & 20 & 15 & 2.5 & 35 & 5 & 1 & 0.5 & 3 \\
\hline
\end{tabular}

According to the normal distribution law, the normalized variable $u_{i}$ is given by (17), we get:

$$
\begin{aligned}
& \frac{y_{i}^{a}-m_{i}}{\sigma_{i}}=-\frac{y_{i}^{b}-m_{i}}{\sigma_{i}}, \quad \text { or } \\
& \frac{y_{i}^{a}-x_{i}}{\sigma_{i}}=-\frac{y_{i}^{b}-x_{i}}{\sigma_{i}}, i=1, \ldots, n .
\end{aligned}
$$

To obtain equality between the reliability indices (see Eq. (16)), the mean value of variable corresponds to the structure at $f_{n}$. So the mean values of safest solution are located in the middle of the variable interval $\left[y_{i}^{a}, y_{i}^{b}\right]$ as follows:

$$
m_{i}=x_{i}=\frac{y_{i}^{a}+y_{i}^{b}}{2}, i=1, \ldots, n .
$$

In a recent publication [10], we found that the safest point method is suitable for modal analysis more than the other methods that are complex to implement and to converge in this kind of study. A numerical application in Section 5.4 shows the efficiency (computing time reduction) of the SP procedure relative to $\mathrm{HM}$ on an aircraft wing under dynamic behavior.

\section{Numerical applications}

\subsection{A bracket: DDO \& RBDO}

The objective of this example is to demonstrate the advantage of the RBDO procedure relative to the DDO one. Here, we minimize the volume of the bracket structure which is illustrated in Figure 5 subject to stress constraint and the target reliability index constraint. This structure is supported at its two upper holes of radius $R 1=2 \mathrm{~mm}$ and loaded at its lower hole of radius $R 2=2.5 \mathrm{~mm}$ by a pressure $P=150 \mathrm{MPa}$. The material is steel with Young's modulus $E=210 \mathrm{GPa}$ and yield stress $\sigma_{y}=235 \mathrm{MPa}$. The target reliability index must be 3.8. Table 1 shows the different parameters of this structure.

To optimize the structure, the mean values $m_{D 1}, m_{D 2}, m_{D 3}, m_{D 5}, m_{D 6}, m_{R 0}$ and $m_{R c}$, of the dimensions D1, D2, D3, D5, D6, R0 and $R c$ are the design variables. The physical dimensions $D 1, D 2, D 3$, $D 5, D 6, R 0$ and $R c$, are elements of the vector of random variables and assumed to be normally distributed. The standard-deviations are given by $\sigma_{D 1}=1.5, \sigma_{D 2}=$ $1.02, \sigma_{D 3}=0.24, \sigma_{D 5}=2.3, \sigma_{D 6}=0.13, \sigma_{R 0}=0.08$ and $\sigma_{R c}=0.1 \mathrm{~mm}$. During the subsequent design optimization processes, we consider all variables to be bounded by upper and lower limits. 


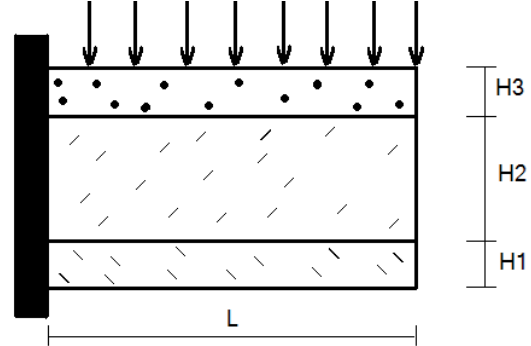

a

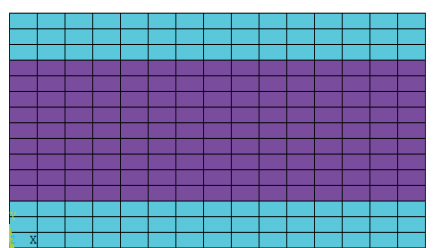

b

Fig. 6. Layout of tri-material cantilever beam.

\subsubsection{DDO procedure}

The objective is to minimize the volume subject to the design constraints and consider a safety factor that is applied to the load and based on engineering experience. The optimization problem is written as:

$$
\begin{aligned}
& \min _{D 1, \ldots, R c}: \operatorname{Volume}(D 1, \ldots, R c) \\
& \text { subject to }: \sigma_{\max }(D 1, \ldots, R c) \leqslant \sigma_{y} / S_{f} \\
& \text { and }: \sigma_{\min }(D 1, \ldots, R c) \geqslant-\sigma_{y} / S_{f}
\end{aligned}
$$

and the corresponding reliability evaluation as:

$$
\begin{aligned}
\min _{u_{D 1}, \ldots, u_{R c}} & : \operatorname{dis}=\sqrt{u_{D 1}^{2}+\ldots+u_{R c}^{2}} \\
\text { subject to } & : \sigma_{y}-\sigma_{\max }\left(u_{D 1}, \ldots, u_{R c}, D 1, \ldots, R c\right) \leqslant 0 \\
& :-\sigma_{y}-\sigma_{\min }\left(u_{D 1}, \ldots, u_{R c}, D 1, \ldots, R c\right) \leqslant 0 .
\end{aligned}
$$

In equation (20), $\sigma_{\max }$ and $\sigma_{\min }$ refer to the most critical points in terms of the maximum and minimum values of the von Mises stress, and we consider three cases for the global safety factor applied to the distributed pressure $P\left(S_{f}=1.5,1.2,1.1\right)$ but this is not the case for equation (21).

After having optimized the structure, the resulting volumes are $V_{D D O}^{S f=1.5}=563.40, V_{D D O}^{S f=1.2}=502.63$ and $V_{D D O}^{S f=1.1}=434.75 \mathrm{~mm}^{3}$ and correspond to reliability indices $\beta_{D D O}^{S f=1.5}=5.73, \beta_{D D O}^{S f=1.2}=4.84$ and $\beta_{D D O}^{S f=1.1}=2.54$ respectively. This way we cannot control the required reliability level (higher or smaller than the required reliability index $\left.\beta_{t}=3.8\right)$, however, by integration of the reliability concept into the optimization process (RBDO case), we can satisfy the reliability constraint.

\subsubsection{HM procedure}

It has been demonstrated that the classical method implies very high computational cost and exhibits weak convergence stability (see Kharmanda et al. [2]). So we use the Hybrid Method (HM) as a flexible RBDO method to satisfy the required reliability level (with admissible tolerances $1 \%$ ). To perform the hybrid RBDO problem, we can write as follows:

$$
\begin{array}{ll}
\min _{m_{D 1}, \ldots, m_{R c}, D 1, \ldots, R c} & : \operatorname{Volume}(D 1, \ldots, R c) \cdot d_{\beta} \\
& \left(m_{D 1}, \ldots, m_{R c}, D 1, \ldots, R c\right. \\
\text { subject to } & : \sigma_{\max }(D 1, \ldots, R c) \leqslant \sigma_{y} \\
& : \sigma_{\min }(D 1, \ldots, R c) \geqslant-\sigma_{y} \\
\text { and } & : d_{\beta}\left(m_{D 1}, \ldots, m_{R c}, D 1,\right. \\
& \ldots, R c) \geqslant \beta_{t} .
\end{array}
$$

The main advantage of the RBDO model relative to the DDO one is that the reliability requirements can be automatically satisfied, but when using the DDO procedure, the designer has to change manually the structure parameters to satisfy the target reliability index. So we consider the hybrid RBDO as an efficient tool to control the structural reliability levels. But the DDO may lead to high or low reliability levels because it does not control the reliability.

\subsection{A tri-material cantilever beam: RBDO for static analysis}

The objective of the following example is to show the advantages of the Optimum Safety Factor (OSF) strategy relative to the Hybrid Method (HM) applied to a compound tri-material beam. We first show that both the OSF and hybrid methods can satisfy the required reliability levels. Next, we demonstrate that the OSF method provides the designer with the lowest structural volume for the same reliability levels relative to the HM.

The design problem under consideration pertains to a short tri-material cantilever beam of length $L=100 \mathrm{~mm}$, height $H=50 \mathrm{~mm}$ and width $T=20 \mathrm{~mm}$, which is loaded by a distributed pressure $q=15 \mathrm{~N} / \mathrm{mm}^{2}$. The beam structure is composed of three layers of material (Fig. 6) of different Young's moduli $E_{1}=200 \mathrm{GPa}, E_{2}=100 \mathrm{GPa}$ and $E_{3}=150 \mathrm{GPa}$, Poisson's ratios $\nu_{1}=0.3, \nu_{2}=0.1$ and $\nu_{3}=0.2$, and yield stresses $\sigma_{1}^{y}=48, \sigma_{2}^{y}=18$ and $\sigma_{3}^{y}=42 \mathrm{MPa}$. The heights of the three layers are: $H 1=10 \mathrm{~mm}, H 2=30 \mathrm{~mm}$, and $H 3=10 \mathrm{~mm}$. To optimize the tri-material beam structure, the mean values $m_{H 1}, m_{H 2}$ and $m_{H 3}$ of the heights $H 1, H 2$ and $H 3$ are the design variables. The physical heights $H 1, H 2$ and $H 3$ are elements of the vector of random variables. The target reliability index is taken to be: $\beta_{t}=3$, and the standard-deviations are given by $\sigma_{H 1}=0.1 m_{H 1}$, 
Table 2. Results of DDO procedure for different values of safety factors.

\begin{tabular}{|c|c|c|c|c|c|c|}
\hline \multirow{2}{*}{ Parameter } & \multicolumn{2}{|c|}{$S f=1.5$} & \multicolumn{2}{|c|}{$S f=1.2$} & \multicolumn{2}{|c|}{$S f=1.1$} \\
\hline & Design point & $\begin{array}{l}\text { Optimum } \\
\text { solution }\end{array}$ & Design point & $\begin{array}{c}\text { Optimum } \\
\text { solution }\end{array}$ & Design point & $\begin{array}{l}\text { Optimum } \\
\text { solution }\end{array}$ \\
\hline$D 1(\mathrm{~mm})$ & 15.215 & 14.552 & 15.119 & 14.861 & 15.160 & 14.910 \\
\hline$D 2(\mathrm{~mm})$ & 10.125 & 9.3327 & 10.021 & 9.3502 & 10.008 & 10.208 \\
\hline D3 (mm) & 2.3730 & 1.8710 & 2.3664 & 1.8965 & 2.3775 & 2.4092 \\
\hline$D 5(\mathrm{~mm})$ & 21.004 & 17.153 & 22.029 & 22.155 & 21.994 & 22.996 \\
\hline$D 6(\mathrm{~mm})$ & 1.8632 & 2.4532 & 1.8476 & 2.5404 & 1.8546 & 1.8936 \\
\hline$R c(\mathrm{rad})$ & 1.0688 & 1.4214 & 1.0303 & 1.3042 & 1.0118 & 0.84577 \\
\hline$R 0(\mathrm{~mm})$ & 1.1603 & 1.6066 & 1.1744 & 1.5083 & 1.1752 & 1.3853 \\
\hline Stress (MPa) & 235.05 & 156.73 & 235.10 & 195.89 & 235 & 213.75 \\
\hline $\operatorname{Vol}\left(\mathrm{mm}^{3}\right)$ & \multicolumn{2}{|c|}{563.40} & \multicolumn{2}{|c|}{502.63} & \multicolumn{2}{|c|}{434.75} \\
\hline$\beta$ & \multicolumn{2}{|c|}{5.73} & \multicolumn{2}{|c|}{4.84} & \multicolumn{2}{|c|}{2.54} \\
\hline
\end{tabular}

Table 3. Result of reliability-based design optimization.

\begin{tabular}{cccc}
\hline \multicolumn{2}{c}{ Parameter } & Design point & $\begin{array}{c}\text { Optimum } \\
\text { solution }\end{array}$ \\
\hline D1 & $(\mathrm{mm})$ & 15.994 & 15.129 \\
D2 & $(\mathrm{mm})$ & 10.515 & 11.857 \\
D3 & $(\mathrm{mm})$ & 2.4546 & 2.0451 \\
D5 $(\mathrm{mm})$ & 21.543 & 21.167 \\
D6 $(\mathrm{mm})$ & 1.9851 & 1.8881 \\
Rc $(\mathrm{rad})$ & 0.84454 & 0.90157 \\
R0 $(\mathrm{mm})$ & 1.2200 & 1.6739 \\
Stress (MPa) & 235.07 & 151.75 \\
Volume (mm & \multicolumn{3}{c}{666.02} \\
\multicolumn{3}{c}{$\beta$} \\
\hline \multicolumn{3}{c}{3.8} \\
\hline
\end{tabular}

$\sigma_{H 2}=0.1 m_{H 2}$ and $\sigma_{H 3}=0.1 m_{H 3}$. During the subsequent design optimization processes, we consider all variables to be bounded by upper and lower limits.

\subsubsection{HM procedure}

In the HM, we minimize the product of the volume and the reliability index subject to the limit state functions and the required reliability level. The RBDO problem is written as

$$
\begin{array}{ll}
\min _{m_{H 1}, m_{H 2}, m_{H 3}, H 1, H 2, H 3} & \operatorname{Volume}(H 1, H 2, H 3) \cdot d_{\beta} \\
& \left(m_{H 1}, m_{H 2}, m_{H 3}, H 1, H 2, H 3\right) \\
\text { subject to } & \sigma_{1}^{\max }(H 1, H 2, H 3) \leqslant \sigma_{1}^{y} \\
& \sigma_{2}^{\max }(H 1, H 2, H 3) \leqslant \sigma_{2}^{y} \\
& \sigma_{3}^{\max }(H 1, H 2, H 3) \leqslant \sigma_{3}^{y} \\
& d_{\beta}\left(m_{H 1}, m_{H 2}, m_{H 3},\right. \\
& H 1, H 2, H 3) \geqslant \beta_{t} .
\end{array}
$$

This optimization process is carried out in a hybrid design space. The resulting optimal values of the reliability index are found to be: $d_{\beta}=3.0001 \approx \beta_{t}$ (i.e., $0.03 \%$ higher than the target reliability index). The resulting optimum volume is determined as: $\mathrm{Vol}^{*}=41782 \mathrm{~mm}^{3}$. The experience of the designer on finite element software plays a very important role in improving the objective
Table 4. Safety factor values.

\begin{tabular}{ccccc}
\hline Var & $\partial \sigma_{1} / \partial y_{i}$ & $\partial \sigma_{2} / \partial y_{i}$ & $\partial \sigma_{3} / \partial y_{i}$ & $S_{f}^{\beta=3}$ \\
\hline$H 1$ & -1.052 & -0.2160 & -0.7318 & 0.8255 \\
$H 2$ & -0.7452 & -0.2041 & -0.6119 & 0.84582 \\
$H 3$ & -0.8432 & -0.6796 & -0.8271 & 0.81084 \\
\hline
\end{tabular}

function and controlling the convergence. Although the method yields results that satisfy the required reliability level within admissible tolerances, the problem is a complex optimization problem and needs a large number of iterations to converge and improve the value of objective function.

\subsubsection{OSF procedure}

We minimize the volume subject to the design constraints in order to find the coordinates of design point (or MPP). This way the optimization problem is simply written as:

$$
\begin{array}{ll}
\min _{H 1, H 2, H 3} & \operatorname{Volume}(H 1, H 2, H 3) \\
\text { subject to } & \sigma_{1}(H 1, H 2, H 3) \leqslant \sigma_{1}^{y} \\
& \sigma_{2}(H 1, H 2, H 3) \leqslant \sigma_{2}^{y} \\
& \sigma_{3}(H 1, H 2, H 3) \leqslant \sigma_{3}^{y} .
\end{array}
$$

The design point is found to correspond to the maximum von Mises stresses $\sigma_{1}^{\max }=47.335 \mathrm{MPa}, \sigma_{2}^{\max }=$ 17.177 $\mathrm{MPa}$ and $\sigma_{3}^{\max }=41.999 \mathrm{MPa}$, that are almost equivalent to the yield stresses $\sigma_{1}^{y}, \sigma_{2}^{y}$ and $\sigma_{3}^{y}$.

Next, we compute the optimum safety factors using (12) for normal distributions. In this example, the number of the deterministic variables is equal to that of the random ones. During the optimization process, we obtain the sensitivity values of the limit state with respect to all variables. So there is no need for additional computational cost. Table 1 shows the results leading to the values of the safety factors, namely the sensitivity results for the different limit state functions. The optimum solution can be calculated by reevaluating of the model. Table 5 presents the different results of the HM and OSF procedure. In order to demonstrate the efficiency of the 


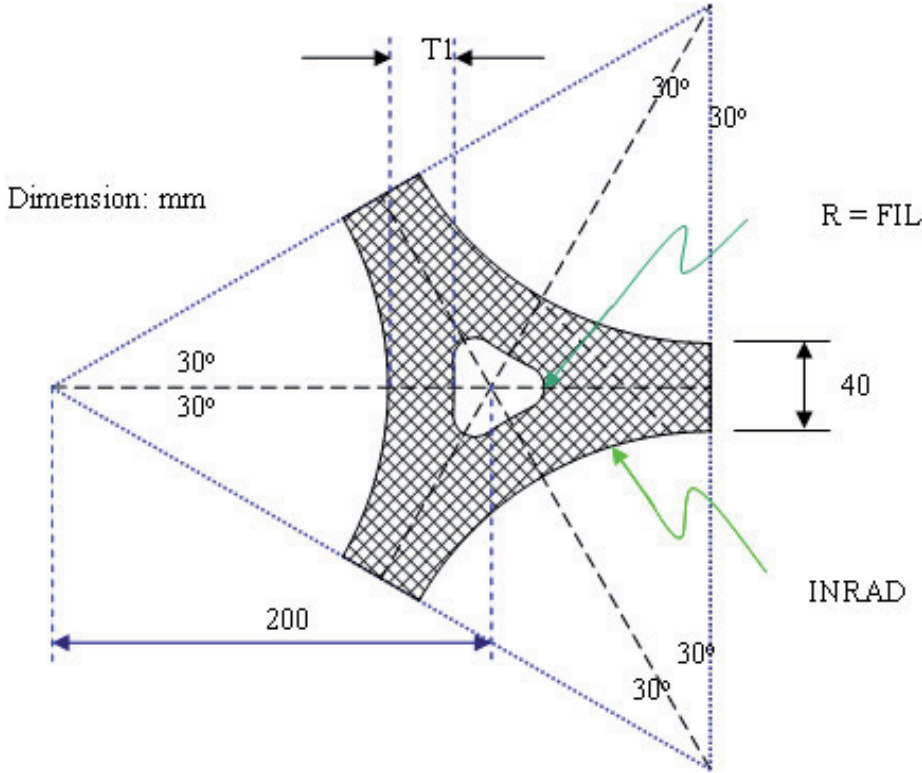

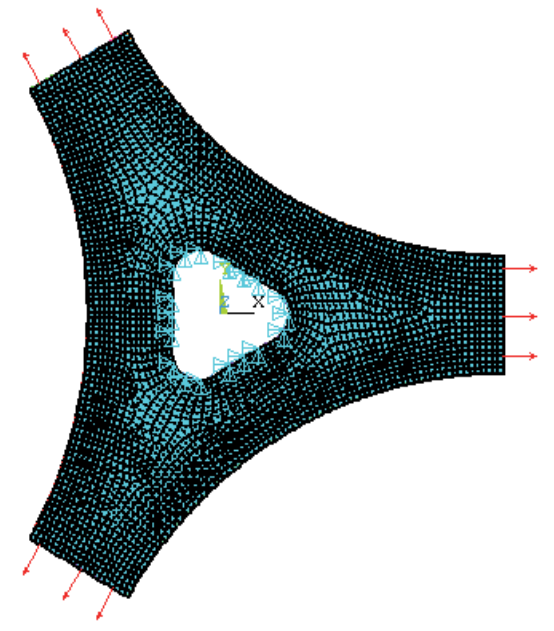

b

Fig. 7. (a) Geometry model and (b) Boudary conditions of triangular plate structure.

Table 5. Results of the OSF and HM.

\begin{tabular}{ccc}
\hline Var & $H M$ & $O S F$ \\
\hline$m_{H 1}$ & 8.3992 & 9.3974 \\
$m_{H 2}$ & 24.753 & 21.851 \\
$m_{H 3}$ & 8.6298 & 9.1176 \\
$\sigma_{1}^{\max }$ & 33.096 & 34.347 \\
$\sigma_{2}^{\max }$ & 12.059 & 12.134 \\
$\sigma_{3}^{\max }$ & 29.718 & 30.915 \\
$H 1$ & 7.4942 & 7.7576 \\
$H 2$ & 18.726 & 18.482 \\
$H 3$ & 7.4368 & 7.3930 \\
$\sigma_{1}^{y}$ & 47.488 & 47.335 \\
$\sigma_{2}^{y}$ & 17.075 & 17.177 \\
$\sigma_{3}^{y}$ & 41.997 & 41.999 \\
$\beta$ & 3.0001 & 3.0000 \\
Vol & 41782 & 40366 \\
\hline
\end{tabular}

OSF method relative to the HM, we can see that the resulting design obtained by the OSF method is better than that obtained by the HM where the objective is to provide the best compromise between cost and safety. The OSF methodology satisfies the required reliability level $\beta_{t}=3$ and gives a smaller structural volume than the HM for the reliability level. In order to improve the resulting structure by the HM, the designer can obtain several local optima and then select the best solution. The resulting optimum volume obtained by OSF $\left(V_{O S F}=40366 \mathrm{~mm}^{3}\right)$ is smaller than the resulting volume determined by the HM by $3.39 \%$.

\subsection{Triangular plate: RBDO for transient analysis}

A triangular plate structure being illustrated in Figure 7a, is submitted to pressure $200 \mathrm{Mpa}$. The Young's modulus is: $207 \mathrm{GPa}$ and Poisson's ratio is: 0.3. The thickness of this plate is: $R 0=10 \mathrm{~mm}$ and $T 1=30 \mathrm{~mm}$. The radius of fillet is: $F I L=10 \mathrm{rad}$. The yield stress is: $\sigma_{y}=235 \mathrm{Mpa}$.

The objective of this study is to show the efficiency and the robustness of the IHM relative to the HM. Here, we can regroup $T 1, R 0$ and $F I L$ in a random vector $\mathbf{y}$ but to optimize the design, the means $m_{T 1}, m_{R 0}$ and $m_{F I L}$ are regrouped in a deterministic vector $\mathbf{x}$, and their fix standard-deviation equals to $0.1 m_{x}$ according the normal distribution. The optimization problem is to find the optimum value of the structural volume subject the maximum stress (transient response).

\subsubsection{HM procedure}

We seek to minimize the hybrid form of the objective function subject to the stress constraint and the reliability one as follows:

$$
\begin{array}{ll}
\underset{T 1, F I L, R 0, m_{T 1}, m_{F I L}, m_{R 0}}{\min } & : \operatorname{Volume}(T 1, F I L, R 0) \cdot d_{\beta} \\
& (T 1, F I L, R 0, \\
& \left.m_{T 1}, m_{F I L}, m_{R 0}\right) \\
& : \sigma_{\max }(T 1, F I L, R 0)-\sigma_{y} \leqslant 0 \\
\text { subject to } & : d_{\beta}\left(T 1, F I L, R 0, m_{T 1},\right. \\
\text { and } & \left.m_{F I L}, m_{R 0}\right) \geqslant \beta_{t} .
\end{array}
$$

\subsubsection{IHM procedure}

We seek to minimize the improved hybrid form of the objective function subject to the stress constraint and the reliability one. This problem can be expressed as: 


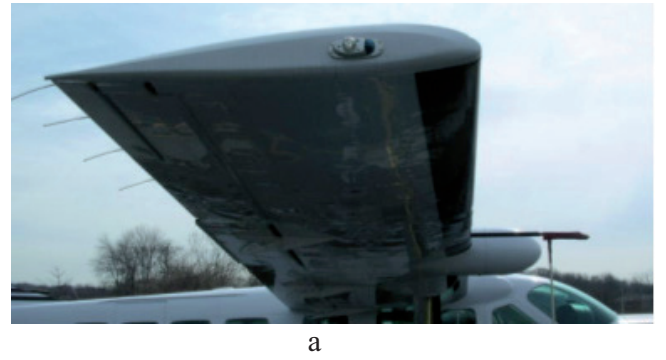

a

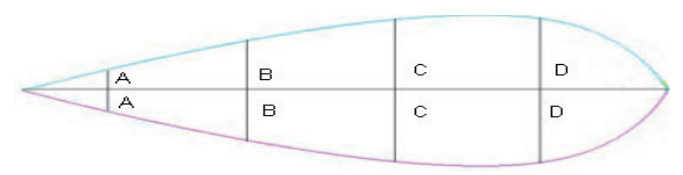

b

Fig. 8. Aircraft wing.

Table 6. Result of HM and IHM.

\begin{tabular}{ccc}
\hline Parameter & HM & IHM \\
\hline$T 1$ & 24.985 & 24.058 \\
$F I L$ & 8.5833 & 9.1013 \\
$R 0$ & 7.3251 & 9.8216 \\
$\sigma_{y}$ & 234.92 & 235.04 \\
$m_{T 1}$ & 29.678 & 26.092 \\
$m_{F I L}$ & 10.600 & 9.1062 \\
$m_{R 0}$ & 7.6991 & 6.0869 \\
$\sigma$ & 204.51366 & 216.420899 \\
Volume & 105874.1 & 78250.5 \\
$\beta$ & 3.8096 & 3.8 \\
\hline
\end{tabular}

$$
\begin{array}{ll}
\min _{T 1, F I L, R 0, m_{T 1}, m_{F I L}, m_{R 0}} & : \operatorname{Vol}\left(T 1, F I L, R 0, m_{T 1},\right. \\
& \left.m_{F I L}, m_{R 0}\right) . \\
& d_{\beta}\left(T 1, F I L, R 0, m_{T 1},\right. \\
& \left.m_{F I L}, m_{R 0}\right) . \\
& \operatorname{Vol}\left(m_{T 1}, m_{F I L}, m_{R 0}\right) \\
& : \sigma_{\max }(T 1, F I L, R 0)-\sigma_{y} \leqslant 0 \\
\text { subject to } & : d_{\beta}\left(T 1, F I L, R 0, m_{T 1},\right. \\
\text { and } & \left.m_{F I L}, m_{R 0}\right) \geqslant \beta_{t}
\end{array}
$$

Table 6 shows the results of both HM and IHM. Both two methods satisfy the required reliability level $\beta_{t} \approx 3.8$. However, the optimal volume obtained by the IHM is less than that obtained by the HM. This way the volume value reduction is almost $26 \%$ that leads to economic structures. This study shows the efficiency and the robustness of the improved hybrid method.

\subsection{Aircraft wing: RBDO for modal analysis}

The wing is uniform along its length with cross sectional area as illustrated in Figure 8a. It is firmly attached to the body of the airplane at one end. The chord of the airfoil has dimensions and orientation as shown in Figure 8b. The wing is made of low density polyethylene with a Young's modulus of $38 \mathrm{e} 3$ psi, Poisson's ration of 0.3 , and a density of $8.3 \mathrm{E}-5 \mathrm{lbf}-\mathrm{sec} 2 / \mathrm{in} 4$. Assume the side of the wing connected to the plane is completely fixed in all degrees of freedom. The wing is solid and material properties are constant and isotropic.

The objective is to find the eigen-frequency for a given interval $[16,18]$, that is located on the safest position of this interval. So $f a=16 \mathrm{~Hz}, f b=18 \mathrm{~Hz}$ and $f n=$ ? Hz, where $f n$ must verify the equality of reliability indices: $\beta_{a}=\beta_{b}$. We can deal with three models: The first structure must be optimized subject to the first frequency value of the given $f a$, the second one must be optimized at the end frequency value of the interval $f b$, and the third structure must be optimized subject to a frequency value fn that verifies the equality of reliability indices relative to both sides of the given interval (see Fig. 8) [10].

\subsubsection{HM procedure}

We minimize the composite form of the objective function subject to the different frequencies constraint and the reliability one as follows:

$$
\begin{array}{ll}
\underset{A_{1}, \ldots, m_{A}, \ldots, A_{2}, \ldots}{\min } & : \operatorname{Vol}_{n}\left(m_{A}, m_{B}, m_{C}, m_{D}\right) . d_{\beta 1}\left(A_{1},\right. \\
& \left.B_{1}, C_{1}, D_{1}, m_{A}, m_{B}, m_{C}, m_{D}\right) . d_{\beta 2} \\
& \left(A_{2}, B_{2}, C_{2}, D_{2}, m_{A}, m_{B}, m_{C}, m_{D}\right) \\
\text { subject to } \quad & : f_{\max }^{1}\left(A_{1}, B_{1}, C_{1}, D_{1}\right)-f_{a} \leqslant 0, \\
& f_{\max }^{2}\left(A_{2}, B_{2}, C_{2}, D_{2}\right)-f_{a} \leqslant 0 \\
\text { and } \quad & d_{\beta 1}\left(A_{1}, B_{1}, C_{1}, D_{1}, m_{A}, m_{B},\right. \\
& \left.m_{C}, m_{D}\right)-d_{\beta 2}\left(A_{2}, B_{2}, C_{2}, D_{2}, m_{A},\right. \\
& \left.m_{B}, m_{C}, m_{D}\right) \leqslant 0 .
\end{array}
$$

\subsubsection{SP procedure}

We have two simple optimization problems:

- The first is to minimize the objective function of the first model subject to the frequency fa constraint as follows:

$$
\begin{array}{ll}
\min & : \operatorname{Vol}_{a}\left(A_{1}, B_{1}, C_{1}, D_{1}\right) \\
\text { subject to } & : f_{\text {max }}^{1}\left(A_{1}, B_{1}, C_{1}, D_{1}\right)-f_{a} \leqslant 0 .
\end{array}
$$

- The second is to minimize the objective function of the second model subject to the frequency fb constraint as follows:

$$
\begin{aligned}
& \min : \operatorname{Vol}_{b}\left(A_{2}, B_{2}, C_{2}, D_{2},\right) \\
& \text { subject to }: f_{\max }^{2}\left(A_{2}, B_{2}, C_{2}, D_{2}\right)-f_{a} \leqslant 0
\end{aligned}
$$


Table 7. Results for the aircraft wing.

\begin{tabular}{|c|c|c|c|c|}
\hline & Variables & Initial & $\begin{array}{l}\text { Optimum design } \\
\text { with SP }\end{array}$ & $\begin{array}{c}\text { Optimum design } \\
\text { with HM }\end{array}$ \\
\hline \multirow{4}{*}{$F N$} & $A$ & 0.13295 & 0.123005 & 0.13526 \\
\hline & $B$ & 0.24112 & 0.228385 & 0.26225 \\
\hline & $C$ & 0.30834 & 0.299635 & 0.29445 \\
\hline & $D$ & 0.26316 & 0.2266 & 0.20797 \\
\hline \multirow{4}{*}{$F A$} & $\overline{A 1}$ & 0.09295 & 0.11300 & 0.13526 \\
\hline & $B 1$ & 0.16112 & 0.21557 & 0.20527 \\
\hline & $C 1$ & 0.22834 & 0.29071 & 0.28851 \\
\hline & $D 1$ & 0.18316 & 0.18443 & 0.21867 \\
\hline \multirow{10}{*}{$F B$} & $A 2$ & 0.17295 & 0.13301 & 0.13999 \\
\hline & $B 2$ & 0.32112 & 0.2412 & 0.25565 \\
\hline & $C 2$ & 0.38834 & 0.30856 & 0.30846 \\
\hline & $D 2$ & 0.34316 & 0.2689 & 0.26600 \\
\hline & $F A$ & 14.056 & 16.000 & 16.025 \\
\hline & $F B$ & 21.744 & 17.913 & 17.960 \\
\hline & $F N$ & 17.800 & 16.85 & 16.94 \\
\hline & $D I F=\beta 1-\beta 2$ & $8.88 E-16$ & $-1.33 E-15$ & $0.60 E-03$ \\
\hline & volume & 6.17144217 & 5.72973783 & 5.84788645 \\
\hline & Time $(S)$ & - & 35.21 & 252.6 \\
\hline
\end{tabular}

and next we compute the coordinates of the third model which corresponds to fn according to equation (19). Table 7 shows the results of the SP method and presents the reliability-based optimum point for a given interval $[16,18]$. The value of $\mathrm{fn}$ is $16.85 \mathrm{~Hz}$, presents the equality of reliability indices. The SP method reduces the computing time by $86 \%$ relative to the hybrid method. So the advantage of the SP method is simple to be implemented on the machine and to define the eigen-frequency of a given interval. It can be also a conjoint of the OSF method.

\section{Conclusion}

A RBDO solution that reduces the structural weight in uncritical regions both provides an improved design and a higher level of confidence in the design. The classical RBDO approach can be carried out in two separate spaces: the physical space and the normalized space. Since very many repeated searches are needed in the above two spaces, the computational time for such an optimization is a big problem. The structural engineers do not consider the RBDO as a practical tool for design optimization. Fortunately, an efficient method called the Hybrid Method (HM) has been elaborated where the optimization process is carried out in a Hybrid Design Space (HDS). The different applications present the flexible uses of the HM that allows us to satisfy a required reliability level for different cases (static, dynamic...). However, the vector of variables here contains both deterministic and random variables. The RBDO problem by HM is thus more complex than that of deterministic design. The major difficulty lies in the evaluation of the structural reliability, which is carried out by a special optimization procedure. Next, an OSF (Optimum Safety Factor) methodology has been proposed to simplify the optimization problem (re- duction of number of variables) and aims to find at least a local optimum solution because it is based on the optimality condition, which is shown in the second example. However, the OSF method cannot be used in some dynamic cases like freely vibrating structures. For example, if the failure interval $[f a, f b]$ is given, we cannot determine the reliability-based optimum solution using the optimum safety factor method. Furthermore, we cannot guarantee even a local optimum when using HM. So there is strong motivation to improve the optimum value of the HM. An Improved Hybrid Method (IHM) has been proposed with object of improving the optimum value of the objective function. The third example shows the power of the IHM to provide the designer with a better optimum value of the objective function relative to the HM. The procedure of IHM is still complex to be implemented on the machine and the OSF method can provide a big part of the RBDO problem analytically (high reduction of the computing time). So since the OSF procedure cannot be used for some special dynamic cases, we here propose a new methodology called Safest Point method (SP) as a conjoint to the OSF method in order to solve the freely vibrating structures as it shown in the last example.

\section{References}

1. A. Der Kiureghian, E. Polak (1998), Reliability-based optimal structural design: A Decoupled Approach, in: Andrzej S. Nowak (ed.), Reliability and Optimization of Structural Systems 197-205

2. O. Ditlevsen, H. Madsen (1996), Structural Reliability Methods. John Wiley \& Sons

3. Y.S. Feng, F. Moses (1986), A method of structural optimization based on structural system reliability. J. Struct. Mech. 14, 437-453

4. R.V. Grandhi, L. Wang (1998), Reliability-based structural optimization using improved two-point adaptive 
nonlinear approximations. Finite Elements in Analysis and Design 29, 35-48

5. A.M. Hasofer, N.C. Lind (1974), An exact and invariant first order reliability format. J. Eng. Mech. 100, 111-121

6. G. Kharmanda, A. Mohamed, M. Lemaire (2002), Efficient reliability-based design optimization using hybrid space with application to finite element analysis. Structural and Multidisciplinary Optimization 24, 233245

7. G. Kharmanda, A. Mohsine, A. El-Hami (2003), Efficient Reliability-Based Design Optimization for Dynamic Structures, In: Proc. Fifth World Congress of Structural and Multidisciplinary Optimization, WCSMO-5, eds. C. Cinquini, M. Rovati, P. Venini, R. Nascimbene, May 1923 (2003), Lido di Jesolo-Venice, Italy. University of Pavia, Italy, 6 pp. (2003)

8. G. Kharmanda, A. El-Hami, N. Olhoff (2004a) Global Reliability-Based Design Optimization, in: Frontiers on Global Optimization, C.A. Floudas, ed., 255 (20), Kluwer Academic Publishers

9. G. Kharmanda, N. Olhoff, A. El-Hami (2004b), Optimum safety factor approach for reliability-based design optimization with extension to multiple limit state case. Structural and Multidisciplinary Optimization, 26

10. G. Kharmanda, A. Altonji, A. Elhami (2006), Safest point method for reliability-based design optimization of freely vibrating structures, CIFMA01-IFCAM01, Aleppo, Syria 02-04 May

11. M. Kleiber, A. Siemaszko, R. Stocki (1999), Interactive stability-oriented reliability-based design optimization. Computer Methods in Applied Mechanics and Engineering 168, 243-253

12. P.-L. Liu, A. Der Kiureghian (1991), Optimization algorithms for structural reliability. Structural Safety 9, $161-177$

13. H.O. Madsen, P. Friis Hansen (1991), Comparison of some algorithms for reliability-based structural optimization and sensitivity analysis, in: Brebbia, C.A. and Orszag, S.A. (eds.): Reliability and Optimization of Structural Systems, pp. 443-451 (Springer-Verlag, Germany)

14. F. Moses (1977), Structural system reliability and optimization. Comput. Struct. 7, 283-290

15. A. Mohsine, G. Kharmanda, A. El-Hami (2005), Reliability-based design optimization study using normal and lognormal distributions with applications to dynamic structures, in: The Fifth International Conference on Structural Safety and Reliability ICOSSAR05, June 19-22 (2005), Rome (Italy)

16. A. Mohsine, G. Kharmanda, A. El-Hami (2006), Improved hybrid method as a robust tool for reliability-based design optimization. Struct. Multidiscipl. Optim. 32, 203$213(2006)$

17. A. Mohsine (2006), Contribution à l'optimisation fiabiliste en dynamique des structures mécaniques, Ph.D. thesis, INSA de Rouen, France (French version)

18. E. Rosenblatt (1976), Optimum Design for Infrequent Disturbances. J. Struct. Div. ASCE 102, ST9, 1807-1825

19. J.D. Stevenson (1967), Reliability analysis and optimum design of structural systems with applications to rigid frames. Division of Solid Mechanics and Structures, 14, Case Western Reserve University, Cleveland, Ohio

20. J. Tu, K.K. Choi, Y.H. Park (1999), A new study on reliability-based design optimization. Journal of Mechanical Design, ASME 121, 557-564

21. R.J. Yang, C. Chuang, L. Gu, G. Li (2005), Experience with approximate reliability-based optimization methods II: an exhaust system problem. Struct. Multidiscipl. Optim. 29, 488-497

22. B.D. Youn, K.K. Choi, Y.H. Park (2003), Hybrid analysis method for reliability-based design optimization. Journal of Mechanical Design 125, 221-232

23. B.D. Youn, K.K. Choi, L. Du (2005), Adaptive probability analysis using an enhanced hybrid mean value method. Struct. Multidiscipl. Optim. 29, 134-148 